\title{
Adaptación y Validación del Cuestionario Factores Contextuales en Países Iberoamericanos
}

\section{Adaptation and Validation of the Contextual Factors Questionnaire in Iberoamerican Countries}

\author{
Laura Lara ${ }^{1}$, Sergio Dominguez-Lara ${ }^{2}$, Juan Miguel Gómez-Espino ${ }^{3}$, Fernando Acevedo ${ }^{4}$, \\ Jorge Alberto Valencia Cobo ${ }^{5}$, Edgardo Miranda-Zapata ${ }^{6}$ y Mahia Saracostti ${ }^{7}$
}

\section{Resumen}

El objetivo de este estudio es adaptar y validar un cuestionario para la medición de los factores contextuales principales (familia, profesores y pares) que influyen en el compromiso escolar de estudiantes adolescentes de Colombia, España, Perú y Uruguay. La equivalencia cultural y lingüística de los ítems que conforman el cuestionario en dichos países se aseguró mediante la revisión de expertos en educación y grupos de discusión. Los cuestionarios fueron aplicados a 1578 estudiantes a través de una plataforma online. Los resultados de los análisis de modelos de ecuaciones estructurales exploratorios indicaron que la estructura en cada uno de los países se adecuaba a la original, con variaciones en los ítems finales.

Palabras clave: compromiso escolar, familia, estudiantes, profesorado

\begin{abstract}
The objective of this study is to adapt and validate a questionnaire to measure the main contextual factors (family, teachers and peers) that influence the school engagement of adolescent students from Colombia, Spain, Peru and Uruguay. The cultural and linguistic equivalence of the items that make up the questionnaire in these countries was ensured through a review by educational experts as well as discussion groups with students. The questionnaires were applied to 1,578 students through an online platform. The results of the exploratory structural equation model analyses indicated that the structure in each country confirmed the original one, with variations in the final items.
\end{abstract}

Keywords: school engagement, family, students, teachers

\footnotetext{
${ }^{1}$ Laura Lara. Doctora en Psicología. Profesora Titular. Universidad Autónoma de Chile, 5 poniente 1670, Talca, Chile. Tel.: +56 712735770. Correo: 1larav@uautonoma.cl.

${ }^{2}$ Doctor en Psicología. Docente-investigador. Universidad de San Martín de Porres y Universidad Privada San Juan Bautista. Av. Juan A Lavalle S/N, Lima 9, Perú. Tel.: +51 2545302. Correo: sergio.dominguez@upsjb.edu.pe

${ }^{3}$ Doctor en Sociología. Profesor Contratado Doctor. Universidad Pablo de Olavide. Facultad de Ciencias Sociales, Universidad Pablo de Olavide de Sevilla, Ctra. de Utrera 1, CP. 41013 Sevilla, España. Tel.: +34 954349348. Correo: jmgomesp@upo.es

${ }^{4}$ Doctor en Educación. Profesor Adjunto. Universidad de la República de Uruguay. Centro Universitario de Rivera, Ituzaingó 667, C.P. 40.000, Rivera, Uruguay. Tel.: +598 99866115. Correo: face@ cur.edu.uy

${ }^{5}$ Magister en Educación. Coordinador Observatorio de Educación de la Universidad del Norte. Universidad del Norte. Km 5 Vía a Puerto Colombia, Barranquilla, Colombia. Tel.: +57 53509115. Correo: javalenciac@ uninorte.edu.co

${ }^{6}$ Doctor en Psicología. Académico e Investigador. Núcleo Científico Tecnológico en Ciencias Sociales y Humanidades, Universidad de La Frontera. Universidad de la Frontera, Avenida Francisco Salazar, 01145. Temuco, Chile. Tel.: + +56 452596732. Correo: edgardo.miranda@ufrontera.cl

${ }^{7}$ Doctora en Social Welfare. Profesora Titular de la Escuela de Trabajo Social. Facultad de Ciencias Sociales. Universidad de Valparaíso. Directora Cátedra UNESCO Niñez y Juventud, Educación y Sociedad. Blanco 951, Valparaíso, Chile. Tel.: +56 998293161. Correo: mahia.saracostti@uv.cl
} 


\section{Introducción}

Los resultados de las investigaciones disponibles coinciden en que el compromiso escolar es una variable muy relevante para el éxito académico, mostrando resultados positivos en aspectos como la retención escolar o las calificaciones obtenidas (Appleton, Christenson, \& Furlong, 2008; Lara et al., 2018). No obstante, este compromiso se encuentra altamente influenciado por los contextos en el que los y las estudiantes se desarrollan. En este sentido, las investigaciones resaltan a la familia, los profesores y los pares como contextos clave de directa influencia sobre el compromiso escolar de los estudiantes (Fernández-Zabala, Goñi, Camino, \& Zulaika, 2016; Miranda-Zapata, Lara, Navarro, Saracostti, \& de-Toro, 2018; Winter, HernándezTorrano, McLellan, Almukhambetova, \& BrownHajdukova, 2020). Existe evidencia que sustenta la asociación entre estos factores contextuales y el compromiso escolar (Estell \& Perdue, 2013), mostrando como el nivel de apoyo que los y las adolescentes sienten en cada uno de estos tres contextos tiene un efecto muy favorable en su compromiso con su aprendizaje (Wang, Henry, \& Degol, 2020). De hecho, existe toda una serie de investigaciones que muestran que estos tres tipos de apoyos se relacionan en general con la satisfacción que los adolescentes sienten en su escuela (Benavente et al., 2018).

Comenzando por la familia, esta se posiciona como un contexto relevante para el desarrollo de trayectorias educativas exitosas (Lara \& Saracostti, 2019; Saracostti et al., 2019). A pesar de que durante la llegada a la adolescencia la familia deja de tener la enorme influencia que tenía en etapas evolutivas anteriores, sigue siendo un contexto importante para el desarrollo de los y las estudiantes, aspecto que se ve reflejado también en el ámbito académico (RodríguezFernández, Droguett, \& Revuelta, 2012). En relación al profesorado, las relaciones que los y las estudiantes mantienen con su profesores y profesoras se constituyen como un factor contextual que ha demostrado tener una especial influencia sobre su compromiso escolar (Brinkworth, McIntyre, Jaraschek, \& Gehlbach, 2018; Martin \& Collie, 2019). De este modo, el proceso de aprendizaje escolar puede concebirse como una experiencia personal y emocional permeada por emociones generadas por la relación entre el estudiante y el profesorado (Neumann, 2009). Finalmente, en relación con el contexto de los pares, durante la adolescencia su influencia adquiere una enorme relevancia, convirtiéndose en uno de los contextos socializadores más relevantes, caracterizado por el deseo de encajar en el grupo (Hamm, Farmer, Lambert, \& Gravelle, 2014). La literatura científica presenta evidencias que sustentan que esta influencia puede ser tanto positiva como negativa para el compromiso escolar de los y las adolescentes, mostrando como los y las estudiantes tienden a ajustar su compromiso a los niveles de su grupo de iguales (Wang, Kiuru, Degol, \& Salmela-Aro, 2018).

A pesar de la evidencia que respalda la influencia de las variables de contexto -familia, profesorado y pares- sobre el compromiso escolar, así como también de forma general sobre los resultados relacionados con el éxito académico, no existe un instrumento para medirlos de forma conjunta. La mayoría de los estudios recurren a escalas diferentes para medir cada uno de estos contextos, no existiendo una escala que permita medir de forma breve la influencia de estos tres contextos sobre el compromiso escolar. Por este motivo, en Chile se ha desarrollado un cuestionario que identifica de forma clara y en un solo instrumento estos tres contextos que influyen sobre el compromiso escolar de los adolescentes (Universidad de la Frontera y Universidad Autónoma de Chile, 2016). En este estudio, el objetivo es adaptar y validar el Cuestionario Factores Contextuales en estudiantes de enseñanza básica y media en Colombia, España, Perú y Uruguay. En estos cuatro países, a pesar de no contar con instrumentos que evalúen de forma conjunta la influencia de estos tres contextos sobre el compromiso escolar, existen evidencias de su incidencia sobre el mismo, haciendo más necesario poder contar con un instrumento validado que permita esta medida. A continuación, de forma breve, comentamos algunos de estos hallazgos.

En Colombia, los análisis sobre los factores asociados al éxito estudiantil muestran que los comportamientos de los docentes -como los refuerzos positivos, la promoción de la 
convivencia, el interés que demuestran por las necesidades de los alumnos o el reconocimiento de sus logros- tienen un efecto positivo sobre el aprendizaje (Martínez, 2010; Pineda et al., 2014). En este sentido, según la información de las pruebas PISA (OCDE, 2019), los y las estudiantes en Colombia mejoran su rendimiento en lectura cuando perciben que el profesorado muestra interés por ellos y ellas. Si bien la familia y los docentes juegan un papel importante en el desarrollo del compromiso escolar, el desempaño académico de los y las estudiantes en Colombia se encuentra altamente influenciado por los/las compañeros y compañeras de clase (Correa \& Orejuela, 2017). De este modo, por ejemplo, los puntajes de los y las estudiantes de educación básica son más altos cuando se sienten aceptados $\mathrm{y}$ aceptadas en el colegio y cuando no son víctimas de agresiones por sus compañeros y compañeras (ICFES, 2011).

En España, como en otros países, el apoyo familiar ha sido frecuentemente traducido en términos de implicación familiar. Sobre esta cuestión, determinados estudios han planteado que la dirección de la causalidad de la implicación no siempre es la que se supone, es decir, no solo los buenos o malos resultados derivan de una alta o baja implicación, sino que también el efecto puede darse se los resultados hacia los modos de implicación, que pueden ser -incluso- boicoteados por el el/la estudiante (Carmona, 2019). Así, pues, la idea de compromiso escolar busca trascender esta idea de la implicación familiar, adoptando una perspectiva que contempla diferentes variables contextuales. Sobre el apoyo escolar del profesorado, si bien existen reiteradas evidencias del efecto del centro escolar en los resultados escolares a través de las buenas (o mejorables) prácticas pedagógicas, de ayuda, de relación o participación activa del alumnado (Flecha \& Cifuentes, 2016), debe considerarse que estas prácticas están mediadas por dinámicas desigualitarias como la segregación escolar, condicionadas, a su vez, por las lógicas de elección/asignación de centro escolar y, en última instancia, por procesos de segregación urbana (Gómez-Espino, 2019). La segregación escolar, capital en las investigaciones que se desarrollan actualmente en España sobre los problemas educativos -y la consiguiente atención a los contextos vulnerables-, está en el centro del programa teórico y empírico del compromiso escolar (en la medida que mediante este concepto se pretenden orientar programas, además, articulados en diversos ejes -incluyendo el estrictamente escolar- que tienen como fin la lucha contra las desigualdades educativas). En tercer lugar, el grupo de iguales (o el efecto compañeros/as) también ha sido objeto de atención como un mecanismo explicativo del efecto del centro escolar, dada la capacidad de arrastre de los y las adolescentes entre sí (en una dirección u otra), y también como criterio básico de elección de centro escolar por parte de los sectores más acomodados (Cordero, Crespo, \& Pedraja, 2013). Considerar esta dimensión en el concepto de compromiso escolar resulta esencial frente a las miradas adulto-céntricas dominantes que relegan la influencia de los iguales (incluso en la percepción de los adultos) a un plano muy secundario.

En Perú, el estudio de los factores contextuales que influyen en el compromiso escolar se ha abordado de forma independiente y focalizando fundamentalmente en el rendimiento académico. Por un lado, existe información sobre la escasa participación de los padres y madres en las labores de educación y cuidado de sus hijos e hijas, muchas veces mediadas por condiciones de pobreza (Sucari, Aza, Anaya, \& García, 2019). En cuanto al profesorado, aunque históricamente su rol ha sido valorado y percibido por el propio docente como influyente (Chuquilin-Cubas, 2014), es uno de los grupos ocupacionales con mayor estrés y carga laboral (Fernández-Arata, Calderón-De la Cruz, Merino-Soto, \& JuárezGarcía, 2020). Esta situación se debe principalmente a las bajas remuneraciones que perciben que dan lugar a que mantengan trabajos paralelos, complicando la conciliación con la vida familiar y social (Díaz, 2015). A la vez, frecuentemente se les atribuye la responsabilidad de los pobres resultados de los y las estudiantes en evaluaciones nacionales e internacionales (UNESCO, 2017). En este panorama, es poco probable que haya una implicación en el desarrollo educativo de los y las estudiantes. Por último, pese a su importancia, la influencia de los pares aún no ha sido estudiada como un factor relevante para el compromiso escolar del 
estudiante, analizándose específicamente como apoyo social, pero sin enfocarlo en el ámbito académico (e.g., Navarro-Loli, Merino-Soto, Dominguez-Lara, \& Lourenço, 2019).

En Colombia, el análisis del compromiso escolar ha estado vinculado de manera estrecha al rendimiento académico y a la permanencia de los y las estudiantes en el sistema educativo (ICFES, 2011; Orjuela, 2012; Pineda-Báez et al., 2014). La evidencia muestra como mayores niveles educativos de padres y madres tiene un efecto positivo sobre la actitud los escolares hacia las actividades académicas, especialmente entre la población con menores niveles socioeconómicos (Correa \& Orejuela, 2017; ICFES, 2011; Ramírez \& Teichler, 2014). De hecho, la evidencia disponible en Colombia pone de manifiesto como las familias más vulnerables desde un punto de vista económico y social son aquellas con menos capacidad para dar soporte a los procesos educativos y con mayor riesgo de fracaso escolar (Guerrero, Cardona, \& Cuevas, 2013; Lastre, López, \& Alcázar, 2018).

En Uruguay la reducción de las tasas de abandono de los estudios en la educación media ha sido una de las principales preocupaciones que dan lugar a las políticas y programas educativos implementados desde mediados de la primera década del presente siglo (Acevedo, 2020). Esas políticas y programas han tenido un efecto democratizador en cuanto a la ampliación del acceso a la educación media, fundamentalmente mediante la promoción de la inclusión educativa de los sectores más vulnerados de la sociedad (Acevedo \& Menni, 2016; De Armas, 2017), pero su impacto no ha sido significativo en el compromiso escolar ni en la calidad de los aprendizajes. Por otra parte, la consideración de dos de las variables contextuales que inciden en el compromiso escolar -el grupo de pares y los cuidadores (madres y padres)- no ha sido motivo de atención especial (Acevedo, 2020), pero el reconocimiento de la influencia del profesorado las modalidades de reclutamiento, su capacitación, su calidad profesional- ocupa un lugar destacado tanto en el ámbito de las políticas públicas como en el de la producción académica (Acevedo, 2011), tal vez porque su abordaje, a diferencia de las variables restantes (externas al "efecto centro"), está más al alcance de la gestión institucional.
A partir de lo expuesto, el objetivo de este estudio es adaptar y validar el Cuestionario Factores Contextuales en estudiantes de enseñanza básica y media procedentes de Colombia, España, Perú y Uruguay. De este modo, se espera que las versiones del cuestionario en cada uno de dichos países presente una estructura de tres factores (familia, profesorado y pares) con adecuados niveles de fiabilidad.

\section{Método}

\section{Participantes}

En el estudio participó un total de 1578 estudiantes que cursaban cursos equivalentes, con edades comprendidas entre los 12 y los 19 años, $55.4 \%$ chicos. En Colombia participaron 438 estudiantes, $55.9 \%$ chicos, con edades comprendidas entre los 12 y los 19 años $(M=15.23, D E=1.66)$ que cursaban $7^{\circ}, 8^{\circ}$ y $9^{\circ}$ de educación básica secundaria y $10^{\circ}$ y $11^{\circ}$ de educación media $(21.7 \%, 17.1 \%, 22.4 \%, 22.1 \%$ y $16.7 \%$, respectivamente). En Uruguay participaron 391 estudiantes, $67.5 \%$ chicos, con edades comprendidas entre los 12 y los 19 años, que cursaban $1^{\circ}, 2^{\circ}$ y $3^{\circ}$ de Ciclo básico de media y $1^{\circ}$ y $2^{\circ}$ de Bachillerato $(20.7 \%, 20.7 \%, 21 \%$, $18.7 \%$ y $18.9 \%$, respectivamente). En España participaron 394 estudiantes, $52.3 \%$ chicos, con edades comprendidas entre los 12 y los 19 años $(M=14.75, D E=1.59)$ que cursaban $1^{\circ}, 2^{\circ}, 3^{\circ}$ y $4^{\circ}$ de Enseñanza Secundaria Obligatoria y $1^{\circ}$ de Bachillerato $(19.8 \%, 20.3 \%, 18.3 \%, 23.6 \%$ y $17.5 \%$, respectivamente). En Perú participaron 355 estudiantes, $45.1 \%$ chicos, con edades comprendidas entre los 12 y los 18 años $(M=14.26, D E=1.38)$ que cursaban $1^{\circ}, 2^{\circ}, 3^{\circ}, 4^{\circ} \mathrm{y}$ $5^{\circ}$ de Educación Secundaria $(18.6 \%, 18.6 \%$, $22.3 \%, 21.1 \%$ y $19.4 \%$, respectivamente).

\section{Instrumentos}

Cuestionario Factores Contextuales (CFC, Universidad de la Frontera y la Universidad Autónoma, 2016), desarrollado para adolescentes chilenos, consta de 18 ítems que se agrupan en tres factores de contexto: familia (3 ítems), profesorado (8 ítems) y pares (7 ítems), con una escala de respuesta tipo Likert de 5 puntos, desde 1 (nunca o casi nunca) a 5 (siempre o casi 
siempre). Los resultados reportados por los autores originales muestran adecuados niveles de ajuste de los datos al modelo de tres factores correlacionados, así como de consistencia interna.

El ajuste y adaptación a otros contextos culturales del CFC se llevó a cabo a través de juicio de expertos y grupos de discusión. Todo este proceso fue llevado a cabo por la misma investigadora, con el objetivo de minimizar posibles sesgos derivados de las diferentes interpretaciones de los ítems, en conjunto con uno de los responsables en cada uno de los países. En todo momento esta adaptación trato de mantener en cada ítem el mismo contenido, solo realizando las modificaciones oportunas para una adecuada comprensión en los contextos culturales.

En cada uno de los países se contó con la colaboración de siete expertos que revisaron el cuestionario y realizaron juicios de adecuación de los ítems, indicando en caso de no adecuación alternativas posibles para su comprensión adecuada por la población objetivo. El proceso seguido fue el siguiente: a) en cada uno de los países se contactó por email a siete expertos y expertas en áreas relacionadas con la educación (psicología de la educación y ciencias de la educación), doctores/as y académicos/as de universidades que trabajan con adolescentes en las edades escolares para las que el cuestionario está dirigido; b) los expertos analizaron individualmente a través un documento creado para tal fin que se les envió vía email; c) una vez recibidas las respuestas de los siete expertos, los cambios sugeridos fueron analizados en conjunto entre la directora alterna del proyecto y la contraparte en cada uno de los países. Como regla, se conservaron de la forma en la que estaban redactados los ítems en los que ningún experto/a señaló ningún problema, mientras que se modificaron directamente aquellos ítems en los que todos los expertos y expertas sugirieron un cambio similar; finalmente, se propusieron provisionalmente cambios en aquellos ítems en los que más de un experto señaló problemas, para ser posteriormente probados en los grupos de discusión presentando tanto la alternativa original como la sugerida por los expertos.

La comprensión adecuada de los cambios propuestos fue analizada mediante la realización de cinco grupos de discusión en cada país (uno por cada grado académico) compuestos por 6 personas cada uno (equiparados en género) con un total de 30 estudiantes en cada uno de los países. Los grupos de discusión se realizaron en los centros educativos donde los y las estudiantes se encontraban matriculados/as, durante la visita de la investigadora a sus respectivos países, con la presencia y participación de al menos la persona responsable en cada país contrapartida.

\section{Procedimiento}

La recogida de los datos se realizó de forma informatizada en los centros educativos participantes de Perú, Colombia, Uruguay y España. La versión adaptada del cuestionario a través de juicios de expertos y grupos de discusión en cada uno de los países participantes fue alojada para cada país en una plataforma informática de forma diferenciada (Saracostti, Lara, \& MirandaZapata, 2019) de manera que cada país podía acceder a la versión del cuestionario de su país. De este modo, el cuestionario fue el mismo (con directa equivalencia entre ítems); solo variaron en algunos casos la redacción y vocabulario, adaptados a cada país, pero manteniendo la equivalencia en el contenido (ver tablas 1, 2, 3 y 4). La plataforma y el apoyo de acceso y demás aspectos relacionados fueron responsabilidad del equipo chileno, que contó con una empresa contratada para tales efectos, a la que se les entregaron los datos necesarios para generar los códigos de acceso.

En cada país los participantes cumplimentaron el cuestionario en los computadores de los centros educativos, durante el horario lectivo. En cada una de las recogidas de datos estuvo presente el responsable en cada uno de los países (o alguna persona que asignaron debidamente capacitada), así como los profesores y/o profesoras del curso para generar un ambiente confortable a los participantes, y como apoyo. En relación a los aspectos éticos, se respetaron todos los propios de una recogida de datos de este calibre, fueron adaptados a los requerimientos de cada país y estuvieron a cargo del investigador responsable en los mismos, contando al menos con la autorización de los centros educativos, los consentimientos de los tutores y los asentimientos de los estudiantes. El estudio recibió la aprobación del Comité de Ética de la Universidad de la 
Tabla 1. Solución factorial muestra colombiana

\begin{tabular}{|c|c|c|c|c|c|c|c|c|c|}
\hline & & FA & PR & PA & ISF & FA & PR & PA & ISF \\
\hline 1 & Hablo con mi familia sobre lo que hago en el colegio & .769 & -.132 & .063 & .948 & .811 & -.192 & .058 & .914 \\
\hline 2 & $\begin{array}{l}\text { Mis padres o acudientes me motivan a trabajar bien en el } \\
\text { colegio }\end{array}$ & .564 & .261 & .034 & .747 & .636 & 208 & .055 & .849 \\
\hline 3 & Cuando tengo un problema, recibo ayuda de mi familia & .568 & .228 & .037 & .796 & .612 & .172 & .001 & .892 \\
\hline 4 & Mis profesores(as) quieren que aprenda mucho & .125 & .748 & -.059 & .951 & .130 & .741 & .132 & .912 \\
\hline 5 & $\begin{array}{l}\text { Cuando tengo un problema, recibo ayuda de algún(a) } \\
\text { profesor(a) }\end{array}$ & .095 & .426 & .155 & .771 & .051 & .464 & .172 & .810 \\
\hline 6 & $\begin{array}{l}\text { Los profesores me motivan a realizar de nuevo una tera si } \\
\text { me equivoco }\end{array}$ & .255 & .419 & .079 & .603 & .196 & .447 & .131 & .677 \\
\hline 7 & $\begin{array}{l}\text { Los profesores se interesan por mí y me ayudan si tengo } \\
\text { dificultades para hacer las tareas }\end{array}$ & .218 & .588 & .001 & .825 & .123 & .616 & .074 & .923 \\
\hline 8 & Me llevo bien con mis profesores & .062 & .765 & .044 & .985 & .053 & .783 & -.02 & .992 \\
\hline 9 & $\begin{array}{l}\text { Mis profesores se preocupan por mí no sólo como } \\
\text { estudiante sino también como persona }\end{array}$ & .068 & .571 & .121 & .917 & -.025 & .640 & .166 & .905 \\
\hline 10 & $\begin{array}{l}\text { En mi colegio los profesores y otros adultos tratan a todos } \\
\text { los estudiantes con respeto }\end{array}$ & 32 & .774 & .019 & .957 & -.151 & .830 & $\begin{array}{c}- \\
.078\end{array}$ & .940 \\
\hline 11 & $\begin{array}{l}\text { En este colegio, se valora la participación y la opinión de } \\
\text { todos(as) }\end{array}$ & -.135 & .696 & .069 & .932 & -.122 & .735 & .041 & .956 \\
\hline 12 & Mis compañeros(as) me apoyan y se preocupan por mí & -.112 & -.124 & .843 & .943 & -.11 & .043 & .813 & .969 \\
\hline 13 & Puedo confiar en mis compañeros(as) & .015 & -.246 & .852 & .887 & .078 & -.137 & .830 & .948 \\
\hline 14 & Mis compañeros(as) del colegio son importantes para mí & -.067 & .309 & .545 & .653 & & & & \\
\hline 15 & Me llevo bien con mis compañeros(as) de curso & -.07 & .419 & .435 & .481 & & & & \\
\hline 16 & $\begin{array}{l}\text { Siento que soy importante para mis compañeros(as) del } \\
\text { colegio }\end{array}$ & -.08 & .025 & .744 & .981 & -.024 & .143 & .641 & .928 \\
\hline 17 & $\begin{array}{l}\text { En mi colegio, por lo menos un(a) compañero(a) me } \\
\text { ayuda en las tareas difíciles }\end{array}$ & .13 & -.027 & .566 & .923 & .113 & .134 & .363 & .717 \\
\hline 18 & Cuando no entiendo algo, mis compañeros me explican & .246 & -.038 & .541 & .752 & & & & \\
\hline
\end{tabular}

Nota. FA=Familia; PR=Profesorado; PA=Pares

Frontera (Chile) y de la Comisión Nacional de Investigación Científica y Tecnológica de Chile.

\section{Análisis de datos}

El análisis de la estructura factorial del cuestionario en cada uno de los países se llevó a cabo mediante moldeamiento exploratorio de ecuaciones estructurales (ESEM, Asparouhov, y Muthen, 2009), realizado de forma separada para cada una de las muestras mediante el programa Mplus v.7 (Muthen \& Muthen, 1998-2015). Se utilizó el método de estimación WLSMV usando las correlaciones policóricas. Se especificó la rotación target oblicua $(\varepsilon=.05$; Asparouhov, \& Muthen, 2009) que consiste en estimar libremente los ítems que pertenecen teóricamente al factor y especificar como cercanos a cero $(\sim)$ a los ítems que no pertenecerían a dicho factor. El modelo fue evaluado en cada país mediante la combinación de los índices de bondad de ajuste del modelo y un enfoque exploratorio para la conservación de los ítems. De este modo, en primer lugar, se consideraron los índices de ajuste CFI y TLI
(>.90; McDonald \& Ho, 2002) y RMSEA (<.08; Jöreskog \& Sörbom, 1993; límite superior del intervalo de confianza <.10; West, Taylor, \& Wu, 2012) y la raíz cuadrada media residual ponderada (WRMR <1; DiStefano, Liu, Jiang, \& Shi, 2018).

En segundo lugar, se procedió con un enfoque exploratorio basado en la conservación de reactivos siguiendo diferentes criterios: a) que el ítem tenga una carga mínima de .40 en su factor teórico; b) que el Índice de Simplicidad Factorial (ISF; Fleming \& Merino, 2005) o el grado en que el ítem es influido predominantemente por un solo factor, sea mayor que .70 (Dominguez-Lara \& Merino-Soto, 2018); y c) en caso de que el ítem tuviera carga factorial mayor que .40 y un ISF aceptable, si la magnitud de las cargas secundarias superan el .3, el ítem se descarta.

Finalmente, la consistencia interna de los cuestionarios fue analizada mediante el alfa de Cronbach, alfa ordinal, y coeficiente omega (>.70; Hunsley \& Marsh, 2008). 
Tabla 2. Solución factorial muestra española

\begin{tabular}{|c|c|c|c|c|c|c|c|c|c|}
\hline & & FA & PR & PA & ISF & FA & PR & PA & ISF \\
\hline 1 & $\begin{array}{l}\text { Hablo con mi familia sobre lo que hago en el } \\
\text { instituto }\end{array}$ & .65 & -.029 & .071 & .979 & .633 & .006 & .042 & .993 \\
\hline 2 & $\begin{array}{l}\text { Mis padres o tutores legales me animan a } \\
\text { trabajar bien en el instituto }\end{array}$ & .686 & .162 & .059 & .912 & .695 & .172 & .043 & .910 \\
\hline 3 & $\begin{array}{l}\text { Cuando tengo un problema, recibo ayuda de mi } \\
\text { familia }\end{array}$ & .873 & -.065 & .029 & .990 & .900 & -.054 & .002 & .995 \\
\hline 4 & Mis profesores/as quieren que aprenda mucho & .115 & .766 & -.057 & .959 & .143 & .745 & -.043 & .942 \\
\hline 5 & $\begin{array}{l}\text { Cuando tengo un problema, recibo ayuda de } \\
\text { algún/a profesor/a. }\end{array}$ & .028 & .741 & .018 & .997 & .046 & .733 & .026 & .992 \\
\hline 6 & $\begin{array}{l}\text { Los profesores/as animan a realizar } \\
\text { nuevamente una tarea si me equivoco }\end{array}$ & -.131 & .913 & -.095 & .954 & -.119 & .893 & -.077 & .963 \\
\hline 7 & $\begin{array}{l}\text { Los profesores/as se interesan por mí y me } \\
\text { ayudan si tengo dificultades para hacer las } \\
\text { tareas }\end{array}$ & -.128 & .949 & -.043 & .970 & -.094 & .920 & -.018 & .984 \\
\hline 8 & Me llevo bien con mis profesores/as & .087 & .385 & .292 & .506 & & & & \\
\hline 9 & $\begin{array}{l}\text { Los profesores/as se preocupan de mí no sólo } \\
\text { como estudiante sino también como persona }\end{array}$ & -.054 & .66 & .144 & .923 & -.039 & .653 & .140 & .903 \\
\hline 10 & $\begin{array}{l}\text { En mi Instituto los profesores/as y otros adultos } \\
\text { tratan a todos los estudiantes con respeto }\end{array}$ & .068 & .677 & -.042 & .979 & .089 & .655 & -.040 & .968 \\
\hline 11 & $\begin{array}{l}\text { En este instituto, se valora la participación y la } \\
\text { opinión de todos/as }\end{array}$ & .032 & .664 & .072 & .979 & .061 & .652 & .076 & .967 \\
\hline 12 & $\begin{array}{l}\text { Mis compañeros/as me apoyan y se preocupan } \\
\text { por mí. }\end{array}$ & -.13 & .02 & .863 & .966 & -.095 & .069 & .826 & .970 \\
\hline 13 & Puedo confiar en mis compañeros/as & -.197 & -.055 & .908 & .928 & -.141 & -.015 & .874 & .962 \\
\hline 14 & $\begin{array}{l}\text { Mis compañeros(as) de instituto son } \\
\text { importantes para mí }\end{array}$ & .019 & -.071 & .849 & .989 & .099 & -.061 & .814 & .970 \\
\hline 15 & Me llevo bien con mis compañeros/as de clase & .163 & .068 & .670 & .903 & .226 & .066 & .631 & .822 \\
\hline 16 & $\begin{array}{l}\text { Siento que soy importante para mis } \\
\text { compañeros(as) de instituto }\end{array}$ & -.059 & -.064 & .860 & .985 & -.01 & -.028 & .823 & .998 \\
\hline 17 & $\begin{array}{l}\text { En mi instituto, al menos un/a compañero/a me } \\
\text { ayuda en las tareas difíciles }\end{array}$ & .29 & .227 & .389 & .307 & & & & \\
\hline 18 & $\begin{array}{l}\text { Cuando no entiendo algo, mis compañeros/as } \\
\text { me lo explican }\end{array}$ & .294 & .185 & .457 & .471 & & & & \\
\hline
\end{tabular}

Nota. FA=Familia; $\mathrm{P}=$ Profesorado; $\mathrm{PA}=$ Pares

\section{Resultados}

De forma separada se realizaron los ESEM por separado con los datos de los cuatro países participantes, y fueron repetidos hasta que la solución factorial cumplió con los criterios explicitados. Los resultados de los análisis, considerando los criterios indicados en el apartado de análisis de datos, dieron lugar a la retención final de 15 ítems para la validación colombiana (Tabla 1), 11 para la validación uruguaya (Tabla 2), 15 para la validación española (Tabla 3) y 17 para la validación peruana (Tabla 4). Los índices de bondad de los modelos fueron adecuados en todos los casos, aunque los RMSEA fueron elevados en el caso de Colombia y España, como se muestra en la Tabla 5.

Finalmente, la fiabilidad fue adecuada en cada uno de los países medida a través de todos los índices analizados (Alfa de Cronbach, Alfa Ordinal y Coeficiente Omega, ver Tabla 6).

\section{Discusión}

A partir del estudio realizado contamos con un cuestionario con evidencias favorables con relación a su estructura interna para medir los factores de contexto que influyen sobre el compromiso escolar en estudiantes en Uruguay, Colombia, Perú y España. Los resultados de los análisis de las propiedades psicométricas mostraron la adecuación de los cuestionarios para cada uno de los países, si bien el RMSEA fue algo elevado en el caso de España y Colombia. La magnitud del RMSEA puede explicarse por un tamaño muestral moderado y por los grados de libertad obtenidos en el modelo (Kenny, Kaniskan, \& McCoach, 2015; Taasoobshirazi \& Wang, 2016), por lo que se aconseja en futuras investigaciones ampliar el tamaño de la muestra.

Los resultados obtenidos brindan la posibilidad de considerar a los tres factores contextuales como una potencial influencia del compromiso escolar de los estudiantes en vista de 
Tabla 3. Solución factorial muestra peruana

\begin{tabular}{|c|c|c|c|c|c|c|c|c|c|}
\hline & & FA & PR & PA & ISF & FA & PR & PA & ISF \\
\hline 1 & Hablo con mi familia sobre lo que hago en el colegio & .524 & .168 & .069 & .842 & .774 & -.134 & .077 & .943 \\
\hline 2 & $\begin{array}{l}\text { Mis padres o apoderados me animan a trabajar bien en } \\
\text { el colegio }\end{array}$ & .491 & .326 & .062 & .587 & .605 & .175 & .021 & .885 \\
\hline 3 & Cuando tengo un problema, recibo ayuda de mi familia & .717 & .257 & -.001 & .835 & .792 & .106 & -.080 & .959 \\
\hline 4 & Mis profesores(as) quieren que aprenda mucho & .178 & .671 & .013 & .903 & .104 & .719 & -.042 & .964 \\
\hline 5 & $\begin{array}{l}\text { Cuando tengo un problema, recibo ayuda de algún(a) } \\
\text { profesor(a) }\end{array}$ & .132 & .647 & -.002 & .941 & .127 & .632 & -.020 & .941 \\
\hline 6 & $\begin{array}{l}\text { Los profesores me animan a realizar nuevamente una } \\
\text { tarea si me equivoco }\end{array}$ & .042 & .605 & .07 & .973 & .064 & .569 & .078 & .954 \\
\hline 7 & $\begin{array}{l}\text { Los profesores se interesan por mí y me ayudan si } \\
\text { tengo dificultades para hacer las tareas }\end{array}$ & .086 & .711 & .081 & .960 & .083 & .695 & .071 & .964 \\
\hline 8 & Me llevo bien con mis profesores & .037 & 627 & .131 & .933 & -.035 & .680 & .111 & .958 \\
\hline 9 & $\begin{array}{l}\text { Los profesores se preocupan de mí no sólo como } \\
\text { estudiante sino también como persona }\end{array}$ & .109 & 685 & .149 & .899 & .092 & .682 & .133 & .920 \\
\hline 10 & $\begin{array}{l}\text { En mi colegio, los profesores y otros adultos tratan a } \\
\text { todos los estudiantes con respeto }\end{array}$ & .016 & .823 & -.063 & .991 & -.172 & .984 & -.122 & .934 \\
\hline 11 & $\begin{array}{l}\text { En este colegio, se valora la participación y la opinión } \\
\text { de todos(as) }\end{array}$ & .063 & .745 & .015 & .989 & -.057 & .804 & -.031 & .991 \\
\hline 12 & Mis compañeros(as) me apoyan y se preocupan por mí & -.129 & .199 & .730 & .857 & .109 & -.002 & .800 & .973 \\
\hline 13 & Puedo confiar en mis compañeros(as) & -.194 & .040 & .764 & .907 & -.062 & -.067 & .835 & .982 \\
\hline 14 & $\begin{array}{l}\text { Mis compañeros(as) del colegio son importantes para } \\
\text { mí }\end{array}$ & -.144 & .126 & .701 & .896 & -.081 & .079 & .744 & .966 \\
\hline 15 & Me llevo bien con mis compañeros(as) de aula & -.109 & .136 & .682 & .908 & -.09 & .130 & .705 & .928 \\
\hline 16 & $\begin{array}{l}\text { Siento que soy importante para mis compañeros(as) } \\
\text { del colegio }\end{array}$ & -.183 & .169 & .787 & .863 & .079 & -.062 & .888 & .981 \\
\hline 17 & $\begin{array}{l}\text { En mi colegio, al menos un(a) compañeros(a) me } \\
\text { apoya con las tareas difíciles }\end{array}$ & .377 & -.259 & .749 & .599 & & & & \\
\hline 18 & $\begin{array}{l}\text { Cuando no entiendo algo, mis compañeros me } \\
\text { explican }\end{array}$ & .299 & -.332 & .872 & .688 & .017 & .036 & .564 & .993 \\
\hline
\end{tabular}

Tabla 4. Solución factorial muestra uruguaya

\begin{tabular}{|c|c|c|c|c|c|c|c|c|c|}
\hline & & FA & PR & $\mathrm{PA}$ & ISF & FA & PR & $\mathrm{PA}$ & ISF \\
\hline 1 & Hablo con mi familia sobre lo que hago en el liceo & .576 & .338 & .057 & .644 & .819 & .080 & .034 & .983 \\
\hline 2 & $\begin{array}{l}\text { Mis padres o tutores me animan a trabajar bien en el } \\
\text { liceo }\end{array}$ & .230 & .527 & .105 & .727 & & & & \\
\hline 3 & Cuando tengo un problema, recibo ayuda de mi familia & .680 & .330 & -.028 & .731 & .719 & .054 & .019 & .991 \\
\hline 4 & Mis profesores(as) quieren que aprenda mucho. & .098 & .761 & .058 & .967 & -.039 & .855 & -.017 & .996 \\
\hline 5 & $\begin{array}{l}\text { Cuando tengo un problema, recibo ayuda de algún(a) } \\
\text { profesor(a). }\end{array}$ & .325 & .514 & .180 & .510 & & & & \\
\hline 6 & $\begin{array}{l}\text { Los profesores me animan a realizar nuevamente una } \\
\text { tarea si me equivoco. }\end{array}$ & -.027 & .748 & .089 & .977 & -.065 & .698 & .072 & .972 \\
\hline 7 & $\begin{array}{l}\text { Los profesores se interesan por mí y me ayudan si } \\
\text { tengo dificultades para hacer las tareas. }\end{array}$ & -.109 & .909 & .036 & .977 & & & & \\
\hline 8 & Me llevo bien con mis profesores. & .145 & .692 & .085 & .917 & .042 & .779 & .003 & .996 \\
\hline 9 & $\begin{array}{l}\text { Los profesores se preocupan por mí no sólo como } \\
\text { estudiante sino también como persona. }\end{array}$ & .316 & .377 & .270 & .192 & & & & \\
\hline 10 & $\begin{array}{l}\text { En mi liceo, los profesores y otros adultos tratan a } \\
\text { todos los estudiantes con respeto. }\end{array}$ & .215 & .649 & .038 & .852 & .073 & .740 & .010 & .985 \\
\hline 11 & $\begin{array}{l}\text { En este liceo, se valora la participación y la opinión de } \\
\text { todos(as). }\end{array}$ & .186 & .680 & .043 & .892 & .114 & .724 & -.026 & .962 \\
\hline 12 & Mis compañeros(as) me apoyan y se preocupan por mí. & .205 & -.210 & .845 & .839 & .162 & -.087 & .822 & .929 \\
\hline 13 & Puedo confiar en mis compañeros(as). & .116 & -.331 & .811 & .771 & -.024 & -.138 & .808 & .957 \\
\hline 14 & Mis compañeros(as) del liceo son importantes para mí. & -.033 & -.080 & .826 & .984 & -.216 & .205 & .790 & .813 \\
\hline 15 & Me llevo bien con mis compañeros(as) de clase. & -.008 & .251 & .528 & .741 & & & & \\
\hline 16 & $\begin{array}{l}\text { Siento que soy importante para mis compañeros(as) } \\
\text { del liceo. }\end{array}$ & .097 & -.126 & .803 & .943 & .089 & .027 & .731 & .976 \\
\hline 17 & $\begin{array}{l}\text { En mi liceo, al menos un(a) compañeros(a) me apoya } \\
\text { con las tareas difíciles. }\end{array}$ & -.353 & .424 & .544 & .252 & & & & \\
\hline 18 & $\begin{array}{l}\text { Cuando no entiendo algo, mis compañeros me } \\
\text { explican. }\end{array}$ & -.423 & .425 & .593 & .242 & & & & \\
\hline
\end{tabular}

Nota. FA=Familia; PR=Profesorado; PA=Pares

su importancia en las trayectorias educativas.

Cabe precisar que tradicionalmente solo se ha considerado al profesorado como elemento relevante en el contexto educativo, ya que el logro de aprendizajes de los y las estudiantes se sustenta frecuentemente en la labor docente, dejando en un 
Tabla 5. Índices de bondad de ajuste

\begin{tabular}{lcccc}
\hline & CFI & TLI & $\begin{array}{l}\text { RMSEA } \\
(\text { IC 90\%) }\end{array}$ & WRMR \\
\hline Colombia & .949 & .915 & $.093(.083, .104)$ & .947 \\
Uruguay & .993 & .984 & $.053(.038, .077)$ & .425 \\
España & .962 & .937 & $.098(.087, .110)$ & .793 \\
Perú & .967 & .949 & $.082(.071, .092)$ & .890 \\
\hline
\end{tabular}

Tabla 6. Coeficientes de fiabilidad de países

\begin{tabular}{lcccc}
\hline & Colombia & España & Perú & Uruguay \\
\hline Familia & & & & \\
Alfa de Cronbach & .73 & .77 & .75 & .73 \\
Alfa Ordinal & .72 & .78 & .77 & .74 \\
Coeficiente omega & .73 & .79 & .77 & .74 \\
Profesorado & & & & \\
Alfa de Cronbach & .86 & .89 & .88 & .85 \\
Alfa Ordinal & .86 & .90 & .90 & .87 \\
Coeficiente omega & .86 & .90 & .90 & .87 \\
Pares & & & & \\
Alfa de Cronbach & .75 & .87 & .86 & .83 \\
Alfa Ordinal & .75 & .89 & .89 & .87 \\
Coeficiente omega & .77 & .90 & .89 & .87 \\
\hline
\end{tabular}

segundo plano a la familia. Existe una amplia evidencia sobre la influencia del profesorado sobre el aprendizaje de los y las estudiantes (LealSoto \& Alonso-Tapia, 2017), siendo menor el énfasis que se coloca en la familia. Sin embargo, no considerar a la familia como aspecto relevante, trae como consecuencia inmediata un trabajo desarticulado entre escuela-familia, lo que se ve reforzado por el poco tiempo que tienen los padres y madres para dedicar a la revisión y acompañamiento en las tareas de sus hijos e hijas, quienes tratan de compensar con la compañía de sus pares. En este sentido, como se ha mostrado, existe una amplia evidencia que sustenta la importancia de la vinculación familia escuela para la mejorar de las trayectorias educativas de los estudiantes (Lara \& Saracostti, 2019; Saracostti, Santana, \& Lara, 2020).

En conclusión, contar con un instrumento que permita la evaluación conjunta de estos factores brindará información relevante para enfocar estrategias más efectivas.

\section{Financiamiento}

Este artículo ha recibido el apoyo de los proyectos FONDEF ID14I20078 y FONDEF IT 19 I0012 financiados por la Comisión Nacional de Investigación Científica y Tecnológica CONICYT, Chile

\section{Referencias}

Acevedo, F. (2011). Dicen. Calidad educativa y gobernabilidad en un instituto de formación docente. Montevideo: Erga e omnes Ediciones.

Acevedo, F. (2020). Factores explicativos del abandono de los estudios en la educación superior en contextos socio-académicos desfavorables. Revista Española de Pedagogía, 78(276), 253-269. doi:10.22550/REP78-2-2020-02.

Acevedo, F., \& Menni, G. (2016). Programas de inclusión educativa implementados en la Educación Media pública de Uruguay: Breve estado del arte y una mirada hacia el programa Compromiso Educativo. En Dalla Corte y Carneiro Sarturi (Eds.), Politicas Públicas da Educação Básica e Superior. Santa Maria: Universidade Federal de Santa Maria (RS, Brasil).

Appleton, J. J., Christenson, S. L., \& Furlong, M. J. (2008). Student engagement with school: Critical conceptual and methodological issues of the construct. Psychology in the Schools, 45(5), 369-386. doi:10.1002/pits.20303

Asparouhov, T., \& Muthén, B. (2009). Exploratory structural equation modeling. Structural Equation Modeling: A Multidisciplinary Journal, 16, 397-438. doi:10.1080/10705510903008204

Benavente, M. V., Cova, F., Pérez- Salas, C. P., Varela, J. J., Alfaro, J., \& Chuecas, J. (2018). Propiedades psicométricas de la Escala Breve de Bienestar Subjetivo en la Escuela para Adolescentes (BASWBSS) en una muestra de adolescentes chilenos. Revista Iberoamericana de Diagnóstico y Evaluación - e Avaliação Psicológica, 48(3), 53-65.

Brinkworth, M. E., McIntyre, J., Juraschek, A. D., \& Gehlbach, H. (2018). Teacher-student relationships: The positives and negatives of assessing both perspectives. Journal of Applied Developmental Psychology, 55, 2438. doi:10.1016/j.appdev.2017.09.002

Carmona, C. A. (2019). Implicación parental, capital cultural y trayectoria filial. Prácticas y estrategias discursivas. Revista Internacional de Sociología, 77(3), 132.

doi:10.3989/ris.2019.77.3.18.024 
Chuquilin-Cubas, J. (2014). Ser profesor de educación secundaria en el Perú: Última opción, un paso transitorio y una oportunidad de empleo. Revista Educación, 38(2), 63-87.

Cordero Ferrera, J. M., Crespo Cebada, E., \& Pedraja Chaparro, F. (2013). Rendimiento educativo y determinantes según PISA: Una revisión de la literatura en España. Revista de Educación, 362, 273-297. doi:10.4438/1988-592X-RE-2011-362-161

Correa, J. B., \& Orejuela, C. (2017). Incidencia de los factores del contexto en el desempeño en matemáticas de los estudiantes de básica secundaria en Colombia. Semestre Económico, 20(44), 159-188. doi:10.22395/seec.v20n44a8

De Armas, G. (2017). Trayectorias educativas en Uruguay. Principales rasgos, tendencias y desafíos para las políticas públicas. Montevideo: UNICEF. Recuperado de https://www.bibliotecaunicef.uy/doc_num. php?explnum_id=184

DiStefano, C., Liu, J. Jiang, N., \& Shi, D. (2018). Examination of the Weighted Root Mean Square Residual: Evidence for trustworthiness?. Structural Equation Modeling: A Multidisciplinary Journal, 25(3), 453-466. doi:10.1080/10705511.2017.1390394

Díaz, H. (2015). Formación docente en el Perú. Realidad y tendencias. Lima: Fundación Santillana.

Dominguez-Lara, S., \& Merino-Soto, C. (2018). Estructura interna del BFI-10P y BFI-15P: un estudio complementario con enfoque CFA y ESEM. Revista Argentina de Ciencias del Comportamiento, 10(3), 22-34. doi:10.32348/1852.4206.v10.n3.21037

Estell, D. B., \& Perdue, N. H. (2013). Social support and behavioral and affective school engagement: The effects of peers, parents, and teachers. Psychology in the Schools, 50(4), 325-339. doi:10.1002/pits.21681

Fernández-Arata, M., Calderón-De la Cruz, G. A., Merino-Soto, C., \& Juárez-García, A. (2020). Efectos sociodemográficos multivariados sobre la experiencia del burnout en docentes de colegios peruanos. Revista Argentina de Ciencias del Comportamiento, 12(1), 40-49.

Fernández-Zabala, A., Goñi, E., Camino, I., \& Zulaika, L. M. (2016). Family and school context in school engagement. European Journal of Education and Psychology, 9(2), 47-55. doi:10.1016/j.ejeps.2015.09.001

Flecha, R., \& Cifuentes, P. Á. (2016). Comunidades de aprendizaje. Padres y Maestros/Journal of Parents and Teachers, (367), 5. Recuperado de https://revistas.comillas.edu/index.php/padres ymaestros/article/view/7114

Fleming, J., \& Merino, C. (2005). Medidas de simplicidad y ajuste factorial: Un enfoque para la construcción y revisión de escalas derivadas factorialmente. Revista de Psicología, 23(2), 252-266.

Gómez-Espino, J. M. (2019). Zonificación escolar, proximidad espacial y segregación socioeconómica: Los casos de Sevilla y Málaga. Revista Española de Investigaciones Sociológicas, 168, 35-54. doi:10.5477/cis/reis.168.35

Guerrero, C., Cardona, Á., \& Cuevas, J. (2013). Factores de riesgo asociados a bajo rendimiento académico en escolares de Bogotá. Investigaciones Andina, 15(26), 654666.

Hamm, J. V., Farmer, T. W., Lambert, K., \& Gravelle, M. (2014). Enhancing peer cultures of academic effort and achievement in early adolescence: Promotive effects of the SEALS intervention. Developmental Psychology, 50(1), 216-228. doi:10.1037/a0032979

Hunsley, J., \& Marsh, E.J. (2008). Developing criteria for evidence-based assessment: An introduction to assessment that work. En J. Hunsley y E. J. Marsh (Eds.), A guide to assessments that work (pp. 3-14). Oxford: Oxford University Press.

Instituto Colombiano para la Evaluación de la Educación-ICFES- (2011). SABER 5o. y 9o. 2009 Síntesis de resultados de factores asociados. Estudios sobre calidad de la educación en Colombia. Recuperado de https://www.icfes.gov.co/documents/20143/2 34129/Informe+Saber $+5+y+9+2009+$ Sintesis + de+resultados+de+factores+asociados +2011 . pdf

Jöreskog, K. G., \& Sörbom, D. (1993). LISREL 8: Structural equation modeling with the SIMPLIS command language. Scientific 
Software International; Lawrence Erlbaum Associates, Inc.

Kenny, D. A., Kaniskan, B., \& McCoach, D. B. (2015). The performance of RMSEA in models with small degrees of freedom. Sociological Methods \& Research, 44(3), 486-507. doi:10.1177/0049124114543236

Lara, L., \& Saracostti, M. (2019). Effect of parental involvement on children's academic achievement in Chile. Frontiers in Psychology, 10, 1-5. doi:10.3389/fpsyg.2019.01464

Lara, L., Saracostti, M., Navarro, J.-J., de-Toro, X., Miranda-Zapata, E., Trigger, J., M., \& Fuster, J. (2018). Compromiso escolar: Desarrollo y validación de un instrumento. Revista Mexicana de Psicología, 35(1), 52-62.

Lastre, K., López L. D., \& Alcázar, C. (2018). Relación entre apoyo familiar y el rendimiento académico en estudiantes colombianos de educación primaria. Psicogente, 21(39), 102-115. doi:10.17081/psico.21.39.2825

Leal-Soto, F., \& Alonso-Tapia, J. (2017). Cuestionario de clima motivacional de la clase: Validez intercultural, intergénero, evolutiva y predictiva. Revista Iberoamericana de Diagnóstico y Evaluación - e Avaliação Psicológica. RIDEP, 45(3), 5770. doi:10.21865/RIDEP45.3.05

Martin, A. J., \& Collie, R. J. (2019). Teacherstudent relationships and students' engagement in high school: Does the number of negative and positive relationships with teachers matter?. Journal of Educational Psychology, 111(5), 861-876. doi:10.1037/edu0000317

Martínez, A. (2010). Un análisis del efecto de pares sobre el rendimiento académico para Colombia. ICFES.(2012) Estudios sobre calidad de la educación en Colombia. Bogotá. Recuperado de https://www2.icfes.gov.co/documents/20143/ 233912/Un+analisis+del+efecto+pares+sobre +rendimiento+academico+para+colombia+PI SA.pdf

McDonald, R. P., \& Ho, M.-H. R. (2002). Principles and practice in reporting structural equation analyses. Psychological Methods, 7(1), 64-82. doi:10.1037/1082-989X.7.1.64
Miranda-Zapata, E., Lara, L., Navarro, J.-J., Saracostti, M., \& de-Toro, X. (2018). Modelización del efecto del compromiso escolar sobre la asistencia a clases y el rendimiento escolar. Revista de Psicodidáctica, 23(2), 102-109. doi:10.1016/j.psicod.2018.02.003

Muthén, L. K., \& Muthén, B. O. (1998-2015). Mplus User's guide (7th ed.). Los Angeles, CA: Muthén \& Muthén.

Navarro-Loli, J. S., Merino-Soto, C., DominguezLara, S., \& Lourenço, A. (2019). Estructura interna de la Multidimensional Scale of Perceived Social Support (MSPSS) en adolescentes peruanos. Revista Argentina de Ciencias del Comportamiento, 11(1), 38-47. doi:10.32348/1852.4206.v11.n1.21532

Neumann, A. (2009). Professing to learn: Creating tenured lives and careers in the American research university (No. 475). JHU Press.

Organización de las Naciones Unidas para la Educación, la Ciencia y la Cultura, UNESCO. (2017). Revisión de las políticas públicas del sector educación en el Perú. Francia: UNESCO. Recuperado de https://unesdoc.unesco.org/ark:/48223/pf0000 260875

Organización para la Cooperación y el Desarrollo Económicos, OCDE. (2019). Resultados evaluación Internacional de Alumnos (PISA) 2018: Colombia. OCDE. Recuperado de https://www.oecd.org/pisa/data/2018databa se/

Orjuela, J. (2012). Determinantes individuales de desempeño en las pruebas de Estado para la educación media en Colombia. ICFES, Estudios sobre la calidad de la educación en Colombia. Bogotá: Ministerio de Educación Nacional. Recuperado de https://www.icfes.gov.co/documents/20143/2 33983/Determinantes+individuales+desempen o+pruebas+estado+para+educacion+media+e n+colombia.pdf

Pineda, C., Bermúdez, J., Rubiano, A., Pava, N., Suárez, R., \& Cruz, F. (2014). Compromiso estudiantil y desempeño académico en el contexto universitario colombiano. RELIEVE. Revista Electrónica de Investigación y Evaluación Educativa, 20(2), 1-20. 
doi:10.7203/relieve.20.2.4238

Ramírez, C., \& Teichler, U. (2014). Factores socioeconómicos y educativos asociados con el desempeño académico, según nivel de formación y género de los estudiantes que presentaron la prueba SABER PRO 2009. Bogotá: Icfes, Estudios sobre calidad de la educación en Colombia.

Rodríguez-Fernández, A., Droguett, L., \& Revuelta, L. (2012). School and personal adjustment in adolescence: The role of academic self-concept and perceived social support. Revista de Psicodidáctica, 17(2), 397-414. doi:10.1387/Rev.Psicodidact.3002

Saracostti, M., Lara, L., Martella, D., Miranda, H., Miranda-Zapata, E., \& Reininger, T. (2019). Influence of family involvement and children's socioemotional development on the learning outcomes of Chilean students. Frontiers in Psychology, 10, 1-7. doi:10.3389/fpsyg.2019.00335

Saracostti, M., Lara, L., \& Miranda-Zapata, E. (2019). Technological platform for online assessment and report generation of school engagement and contextual factors: Brief technical report. Electronic Journal of Research in Educational Psychology, 17, 193212. doi:10.25115/ejrep,17i47.2049

Saracostti, M., Santana, A., \& Lara, L. (2019). La relación entre familias y escuelas en Chile: Aprendizajes desde la política educativa, la investigación $\quad y \quad$ la intervención socioeducativa. Santiago de Chile: RIL Editores.

Sucari, W., Aza, P., Anaya, J., \& García, J. (2019). Participación familiar en la educación escolar peruana. Revista Innova Educación, 1(1), 6-18. doi:https://doi.org/10.35622/j.rie.01.001

Taasoobshirazi, G., \& Wang, S. (2016). The performance of the SRMR, RMSEA, CFI, and TLI: An examination of sample size, path size, and degrees of freedom. Journal of Applied Quantitative Methods, 11(3), 31-39.

Universidad de la Frontera \& Universidad Autónoma de Chile. (2016). Cuestionarios de evaluación de Compromiso Escolar y Cuestionarios de Factores Predictores, Contextuales y/o Individuales de Compromiso
Escolar. Chile, 270.853. Departamento de Derechos Intelectuales.

Wang, M.-T., Henry, D. A., \& Degol, J. L. (2020). Chapter four: A development-insociocultural context perspective on the multiple pathways to youth's engagement in learning. Advances in Motivation Science, 7, 113-160. doi:10.1016/bs.adms.2019.11.001

Wang, M.-T., Kiuru, N., Degol, J. L., \& SalmelaAro, K. (2018). Friends, academic achievement, and school engagement during adolescence: A social network approach to peer influence and selection effects. Learning and Instruction, 58, 148-160. doi:10.1016/j.learninstruc.2018.06.003

West, S. G., Taylor, A. B., \& Wu, W. (2012). Model fit and model selection in structural equation modeling. En R. H. Hoyle (Ed.), Handbook of Structural Equation Modeling (pp. 209-231). New York: The Guilford Press.

Winter, L., Hernández-Torrano, D., McLellan, R., Almukhambetova, A., \& Brown-Hajdukova, E. (2020). A contextually adapted model of school engagement in Kazakhstan. Current Psychology. doi:10.1007/s12144-020-00758-5 